\title{
Qualitative counting closed geodesics
}

\author{
Bastien Karlhofer ${ }^{1} \cdot$ Jarek Kędra $^{2} \mathbb{D} \cdot$ Michał Marcinkowski $^{3} \cdot$ Alexander Trost $^{1}$
}

Received: 22 May 2019 / Accepted: 2 January 2021 / Published online: 28 January 2021

(c) The Author(s) 2021

\begin{abstract}
We investigate the geometry of word metrics on fundamental groups of manifolds associated with the generating sets consisting of elements represented by closed geodesics. We ask whether the diameter of such a metric is finite or infinite. The first answer we interpret as an abundance of closed geodesics, while the second one as their scarcity. We discuss examples for both cases.
\end{abstract}

Mathematics Subject Classification 20F65 · 53C22

\section{Introduction}

It is a classical observation due to John Milnor [12] and Albert Schwarz [13] that the word metric on the fundamental group of a closed manifold carries information about the Riemannian metric of the universal cover (the metrics are quasi-isometric). In this approach the word metric on the fundamental group is associated with a finite generating set. In the present paper we explore the word metrics on the fundamental group associated with geometrically meaningful generating sets. Specifically, we consider generating sets consisting of closed local geodesics. We then ask the most basic question as to whether the diameter of such a word metric is finite or infinite. The first answer is interpreted as abundance of closed geodesics while the second as their scarcity. We present examples for both cases.

\footnotetext{
Jarek Kędra

kedra@abdn.ac.uk

Bastien Karlhofer

r01bdk17@abdn.ac.uk

Michał Marcinkowski

marcinkow@math.uni.wroc.pl

Alexander Trost

r01aat17@abdn.ac.uk

1 University of Aberdeen, Aberdeen, UK

2 University of Aberdeen and University of Szczecin, Aberdeen, UK

3 IMPAN, Wrocław, Poland
} 


\subsection{Statement of the results}

Let $(M, d)$ be a complete Riemannian manifold and let $C_{x}$ denote the set of closed local geodesics based at $x \in M$. Let $\Gamma_{x} \subseteq \pi_{1}(M, x)$ denote the subgroup of the fundamental group generated by elements represented by closed local geodesics. We are interested in the word norm on $\Gamma_{x}$ associated with the set $S$ of the elements represented by closed geodesics. We call it the closed geodesic norm. We apply methods of geometric group theory to prove the following results.

Theorem 1.2 Let $(M, d)$ be a closed Riemannian manifold of negative curvature admitting a geodesic symmetry through $x \in M$. If $\Gamma_{x}$ is nonabelian then the diameter of the closed geodesic norm is infinite.

The situation changes if the manifold is only non-positively curved. A rich source of examples is provided by locally symmetric spaces $M=\Gamma \backslash G / K$, where $G$ is a semisimple Lie group, $K \subset G$ a maximal compact subgroup and $\Gamma \subset G$ a lattice. The natural metric on $M$ is non-positively curved and we have the following result.

Theorem 1.3 Let $(M, d)$ be a complete Riemannian manifold of nonpositive curvature admitting a geodesic symmetry through $x \in M$. If $\pi_{1}(M, x)$ is isomorphic to a finite index subgroup in an irreducible S-arithmetic Chevalley group of rank at least 2 then the diameter of the closed geodesic norm on $\Gamma_{x}$ is finite.

Our proof of Theorem 1.3 amounts to showing that the closed geodesic norm is bounded above by a conjugation invariant norm and then we use the fact that such norms have finite diameter on S-arithmetic Chevalley groups [7,8]. It would be interesting for find a direct geometric argument which would prove a more general statement.

Conjecture 1.4 Let $M=\Gamma \backslash G / K$ be a locally symmetric space of rank at least 2 . If the lattice $\Gamma$ is invariant under the Cartan involution then the diameter of the closed geodesic norm is finite.

An equivalent form of the above conjecture is that the diameter of the word norm on $\Gamma$ associated with the generating set consisting of elements invariant under the Cartan involution is finite. More generally, it is not known whether conjugation invariant norms on lattices in Lie groups of rank at least 2 have finite diameter. A piece of evidence that their diameter may be finite comes from the fact that such lattices do not admit unbounded quasimorphisms. The above Conjecture 1.4 is a much weaker statement in this direction.

\subsection{A comment on counting closed geodesics}

Classically, counting closed geodesics is done in the form of estimates of the number of geodesics of a given length [1]. Here, we propose a different way of counting. Namely, by measuring how big the subgroup of the fundamental group generated by closed local geodesics is and whether it has finite or infinite diameter with respect to the closed geodesic norm. Finite diameter of the closed geodesic norm is interpreted as abundance of closed local geodesics and infinite diameter as their scarcity. For example, on a flat torus every element of the fundamental group is represented by a closed local geodesic so $\Gamma_{x}=\pi_{1}\left(\mathbf{T}^{n}, x\right)$ and the norm has diameter one. On the other hand, on a hyperbolic punctured torus, we have that $\Gamma_{x}=\pi_{1}\left(\mathbf{T}^{2} \backslash\{0\}\right)=\mathbf{F}_{2}$ for a suitably chosen basepoint and the closed geodesic norm is equivalent to the palindromic length on the free group; see Example 1.7 for details. 


\subsection{Examples}

Example 1.7 (Hyperbolic punctured torus) Let $(M, d)$ be the hyperbolic punctured torus viewed as a quotient of an ideal hyperbolic square. Let the basepoint be represented by the centre of the square. Then the generators of the fundamental group are represented by closed geodesics (drawn in blue in Fig. 1). The central symmetry of the square defines a geodesic symmetry $I: M \rightarrow M$ such that it acts on the fundamental group $\pi_{1}(M, x)=\mathbf{F}_{2}=\langle a, b\rangle$ by inverting generators. It follows that closed local geodesics represent palindromes in the free group $\mathbf{F}_{2}$. Indeed, $I(w(a, b))=w\left(a^{-1}, b^{-1}\right)$ is equal to $w(a, b)^{-1}$ if and only if the reduced word $w(a, b)$ is a palindrome. Thus the closed geodesic norm is equal to the palindromic length on $\mathbf{F}_{2}$ and this is known to have infinite diameter [2], [3, Example 6.7].

Example 1.8 (Hyperbolic closed surface I) Let $\Sigma$ be a closed hyperbolic surface of genus $g$ obtained as a quotient of a regular hyperbolic $4 g$-gon in which the opposite sides are identified and with its centre representing the basepoint. As in the case of the punctured torus the central symmetry defines a geodesic symmetry which is the hyperelliptic involution. Also in this case $\Gamma_{x}=\pi_{1}(\Sigma, x)$. It follows from Theorem 1.2 that the diameter of the closed geodesic norm is infinite.

Example 1.9 (Hyperbolic closed surface II) Let $\Sigma$ be a closed hyperbolic surface of genus 2 obtained as a quotient of a regular hyperbolic octagon with identifications which yield the following presentation of the fundamental group

$$
\pi_{1}(\Sigma, x)=\langle a, b, c, d \mid[a, b][c, d]=1\rangle .
$$

As before the basepoint is represented by the centre of the octagon and its central symmetry descends to a geodesic symmetry $I$ of $\Sigma$. Observe, that the homomorphism induced by $I$ on the first homology is defined by $I_{*}[a]=[c]$ and $I_{*}[b]=[d]$. Thus the subspace of $H_{1}(\Sigma ; \mathbf{R})$ consisting of elements such that $I_{*}(z)=-z$ is 2-dimensional generated by $[a]-[c]$ and $[b]-[d]$. In particular, the subgroup $\Gamma_{x} \subseteq \pi_{1}(\Sigma, x)$ is infinite and of infinite index. It is not difficult to see that is also nonabelian. Thus it follows from Theorem 1.2 that the closed geodesic norm on $\Gamma_{x}$ has infinite diameter.

Example 1.10 (Closed hyperbolic 3-manifold) Let $D \subseteq \mathbf{H}^{3}$ be a right-angled regular hyperbolic dodecahedron and let $W \subset \operatorname{Iso}\left(\mathbf{H}^{3}\right)$ be the right-angled Coxeter group of isometries of the hyperbolic space generated by the reflections in the faces of $D$. Let $\Pi$ be the kernel of the homomorphism $W \rightarrow(\mathbf{Z} / 2 \mathbf{Z})^{6}$ which sends reflections through the opposite faces to the

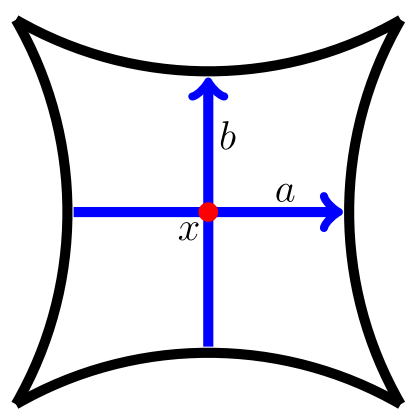

Fig. 1 Punctured torus 
same generator. Then $M=\mathbf{H}^{3} / \Pi$ is a closed hyperbolic manifold glued from $2^{6}$ dodecahedra. Thus $\Pi=\pi_{1}(M, x)$. Moreover, the geodesic symmetry $I: \mathbf{H}^{3} \rightarrow \mathbf{H}^{3}$ at the centre of the dodecahedron $x \in D$ descends to a geodesic symmetry of $M$. To see this observe that if $s \in W$ is a generator then $I s I=s^{\prime}$, where $s^{\prime} \in W$ is the reflection in the face of $D$ opposite to the face of reflection $s$. This means that the conjugation by $I$ preserves $W$ and, moreover, $I s s^{\prime} I=s^{\prime} s=\left(s s^{\prime}\right)^{-1}$. Since conjugates of the six elements $s s^{\prime}$ by elements of $W$ generate $\Pi$ we obtain that the conjugation by $I$ preserves $\Pi$ and that $\Gamma_{x}=\pi_{1}(M, x)$. It follows from Theorem 1.2 that the closed geodesic norm in $\pi_{1}(M, x)$ has infinite diameter.

Example 1.11 Chinburg and Reid [6] proved that there are infinitely many noncomensurable examples of closed hyperbolic 3-manifolds in which all closed geodesics are simple. Let $M$ be such a manifold. It follows that $M$ cannot admit a geodesic symmetry through a point $x \in M$ contained in a closed geodesic. For if $I: M \rightarrow M$ was a geodesic symmetry through a point $x \in \gamma$, where $\gamma$ is a geodesic segment with endpoints at $x$ then $\gamma * I(\gamma)$ would be a closed geodesic with a self-intersection at $x$.

Furthermore, Jones and Reid [9] proved later that if two closed geodesics in $M$ intersect then they are perpendicular. They moreover, proved that $M$ has points at which at least two closed geodesic intersect. Let $x \in M$ be such a point. It follows that at most three closed geodesics can intersect at $x$ for dimensional reasons and hence the group $\Gamma_{x}$ is finitely generated.

Example 1.12 (Locally symmetric space of higher rank) Let $G$ be a non-compact semisimple Lie group, $K \subset G$ its maximal compact subgroup and $\Gamma \subset G$ a lattice. If $I: G \rightarrow G$ is a Cartan involution preserving the lattice (setwise) then it descends to a geodesic symmetry of the locally symmetric space $\Gamma \backslash G / K$.

Let $\Gamma \subseteq \operatorname{SL}(n, \mathbf{Z})$ be a finite index subgroup so that the locally symmetric space $M=$ $\Gamma \backslash \operatorname{SL}(n, \mathbf{R}) / \mathrm{SO}(n)$ is a manifold. The geodesic symmetry is given by the inverse-transpose and hence the closed geodesics represent symmetric matrices of $\Gamma$. If $n=2$ then the space is hyperbolic and the closed geodesic norm has infinite diameter. If $n>2$ then $\operatorname{SL}(n, \mathbf{Z})$ is an arithmetic Chevalley group of rank at least 2 and it follows from Theorem 1.3 that the diameter of the closed geodesic norm is finite. Observe that in this case the group $\Gamma_{x}$ is infinite.

\section{Definitions and supporting results}

\subsection{Geodesics}

We use terminology from [4]. Let $(M, d)$ be a metric space and let $x, y \in M$. A map $\gamma:[a, b] \rightarrow M$ is called a geodesic from $x$ to $y$ if $d(\gamma(s), \gamma(t))=|s-t|$ for every $s, t \in[a, b]$ and $\gamma(a)=x$ and $\gamma(b)=y$. The image of such $\gamma$ is called a geodesic segment. A local geodesic is a map $\gamma:[a, b] \rightarrow M$ such that for every $c \in[a, b]$ there exists an $\epsilon>0$ such that $d(\gamma(s), \gamma(t))=|s-t|$ for every $s, t \in[c-\epsilon, c+\epsilon]$. A metric space $(M, d)$ is called a geodesic metric space if every two points of $M$ can be joined by a geodesic. A complete connected Riemannian manifold is a geodesic metric space.

Let $\left(\mathbf{S}^{1}, g\right)$ denote a circle with the standard metric of total length $2 \pi$. A (locally) isometric embedding $c: \mathbf{S}^{1} \rightarrow M$ is called a closed (local) geodesic. If $\gamma:[a, b] \rightarrow M$ is a path then its reverse $\bar{\gamma}:[a, b] \rightarrow M$ is defined by $\bar{\gamma}(t)=\gamma(a+b-t)$. We define similarly the reverse of a loop $\gamma: \mathbf{S}^{1} \rightarrow M$. A geodesic symmetry $I: M \rightarrow M$ at $x \in M$ is an isometry such that $(I \circ \gamma)(t)=\gamma(-t)=\bar{\gamma}(t)$ for every geodesic $\gamma:[-a, a] \rightarrow M$ such that $\gamma(0)=x$. 
Lemma 2.2 ([4, Theorem 4.13, Chapter II.4]) Let $(M, d)$ be a complete, non-positively curved Riemannian manifold with a basepoint $x \in M$. Then every element $g \in \pi_{1}(M, x)$ is represented by a unique local geodesic $\gamma:[0, a] \rightarrow M$.

Lemma 2.3 ([10, Theorem 3.8.14]) If $(M, d)$ is closed Riemannian manifold of negative curvature then every free homotopy class of loops is represented by a unique closed local geodesic. In particular, each conjugacy class in $\pi_{1}(M, x)$ is represented by a unique closed local geodesic.

\subsection{Quasimorphisms and norms on groups}

Let $G$ be a group. A function $\psi: G \rightarrow \mathbf{R}$ is called a quasimorphism if there exists $D \geq 0$ such that

$$
|\psi(g)-\psi(g h)+\psi(h)| \leq D,
$$

for every $g, h \in G$. The smallest number $D$ with the above property is called the defect of $\psi$. If $\psi\left(g^{n}\right)=n \psi(g)$ for every $n \in \mathbf{Z}$ and every $g \in G$ then $\psi$ is called homogeneous; see [5] for background on quasimorphisms.

A function $v: G \rightarrow \mathbf{R}$ such that for all $g, h \in G:$

- $v(g) \geq 0$,

- $v(g)=0$ if and only if $g=1$,

- $v(g h) \leq v(g)+v(h)$

is called a norm on a group $G$. If in addition $v\left(h g h^{-1}\right)=v(g)$ then $v$ is called conjugation invariant. The supremum $v(G)=\sup \{v(g) \mid g \in G\}$ is called the diameter of $v$ or the diameter of $G$ with respect to $v$. If $v(G)=\infty$ then $v$ is called unbounded.

The number $\tau(g)=\lim _{n \rightarrow \infty} \frac{v\left(g^{n}\right)}{n}$ is called the translation length of $g$ with respect to the norm $v$. If a group $G$ contains an element with positive translation length with respect to the norm $v$ then $v$ is called stably unbounded.

Example 2.5 Let $\Sigma_{\infty}$ be an infinite symmetric group. That is, a group of finitely supported bijections of a countably infinite set. The cardinality of the support defines a conjugation invariant norm of infinite diameter in which every element has translation length equal to zero.

\section{Proofs}

Let $(M, d)$ be a complete Riemannian manifold. Let $I: M \rightarrow M$ be a geodesic symmetry through $x \in M$. By an abuse of notation we denote the induced automorphism of the fundamental group by $I: \pi_{1}(M, x) \rightarrow \pi_{1}(M, x)$. Define the following two subsets of the fundamental group of $M$ :

$$
\begin{aligned}
& S=\left\{g \in \pi_{1}(M, x) \mid I(g)=g^{-1}\right\} \\
& C=\left\{g \in \pi_{1}(M, x) \mid g=[c], c \in C_{x}\right\} .
\end{aligned}
$$

Recall that $C_{x}$ denotes the set of all closed local geodesics through $x \in M$.

Lemma 3.1 If every element of $\pi_{1}(M, x)$ has a unique geodesic representative then $S=C$. 
Proof If $c$ is a closed local geodesic through $x$, then $\bar{c}$ and $I(c)$ have a common initial segment. Thus, by the uniqueness of extension of geodesics, $\bar{c}=I(c)$ and $I[c]=[c]^{-1}$. This proves that $C \subseteq S$.

Let $\gamma$ be a local geodesic representing $s \in S$. Since $I(s)=s^{-1}$ is, on the one hand, represented by a geodesic segment $I \circ \gamma$ and, on the other hand, by a local geodesic $\bar{\gamma}$, we get that $I \circ \gamma=\bar{\gamma}$ due to the uniqueness of geodesic representatives. This implies that $\gamma \in C_{x}$ and hence $s \in C$.

Remark 3.2 Observe that it is important here that $M$ is a manifold. More precisely, that a geodesic is uniquely determined by its initial segment. For example, the graph presented on Fig. 2 admits a geodesic symmetry through $x$ but its closed geodesics going around one of the squares are not preserved setwise.

Lemma 3.3 If every element of $\pi_{1}(M, x)$ has a unique local geodesic representative then the subgroup $\Gamma_{x} \subseteq \pi_{1}(M, x)$ is normal.

Proof According to Lemma 3.1 the subgroup $\Gamma_{x}$ is generated by the set $S$. Let $s \in S$ and let $g \in \pi_{1}(M, x)$. Then

$$
g s g^{-1}=g_{s} I_{x}\left(g^{-1}\right) \cdot I_{x}(g) g^{-1},
$$

which means that the conjugate of an element $s \in S$ is a product of two elements from $S$.

Corollary 3.4 If every element of $\pi_{1}(M, x)$ has a unique local geodesic representative then the closed geodesic norm on $\Gamma_{x}$ is dominated by a norm invariant with respect to the conjugation action of $\pi_{1}(M, x)$.

Proof Let $\bar{S}=\bigcup_{g \in \pi_{1}(M, x)} g S g^{-1}$. Since $\bar{S}$ is invariant under conjugations by elements of $\pi_{1}(M, x)$ the associated word norm is invariant under the conjugation action by $\pi_{1}(M, x)$.

Let $\|g\|_{\bar{S}}=n$. This means that $g=s_{1}^{g_{1}} \cdots s_{n}^{g_{n}}$, where $s_{i} \in S$ and $g_{i} \in \pi_{1}(M, x)$. It follows from Lemma 3.3 that

$$
\|g\|_{S}=\left\|s_{1}^{g_{1}} \cdots s_{n}^{g_{n}}\right\|_{S} \leq 2 n=2\|g\|_{\bar{S}} .
$$

Proof of Theorem 1.3 If $\Gamma_{x}$ is a finite group then there is nothing to prove. So assume that $\Gamma_{x}$ is infinite. Since it is normal in $\pi_{1}(M, x)$, it is of finite index, according to [11, (5.3) Proposition, p.324]. If follows from $[7,8]$ that every conjugation invariant norm on a finite index subgroup of an $\mathrm{S}$-arithmetic Chevalley group of rank at least 2 has finite diameter and hence the statement follows from Corollary 3.4.

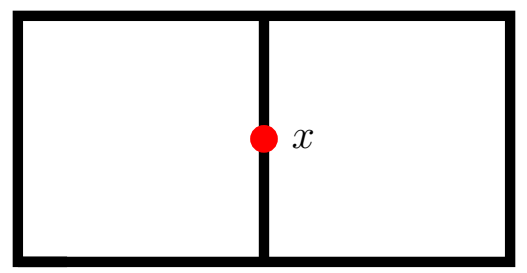

Fig. 2 Warning example 
Proof of Theorem 1.2 Let $\alpha \in \Omega^{1}(M)$ be a differential 1-form and let $\psi_{\alpha}: \pi_{1}(M, x) \rightarrow \mathbf{R}$ be defined by

$$
\psi_{\alpha}(g)=\int_{\gamma} \alpha,
$$

where $\gamma$ is a closed local geodesic representing the conjugacy class of $g$. The map $\psi_{\alpha}$ is a homogeneous quasi-morphism, see [5, Example 2.3.1].

If $\alpha$ is such that $I^{*}(\alpha)=\alpha$ then $\psi_{\alpha}$ vanishes on $S$. Indeed,

$$
\begin{aligned}
\psi_{\alpha}(s) & =\int_{c} \alpha=\int_{I \circ \bar{c}} \alpha \\
& =\int_{\bar{c}} I^{*}(\alpha)=-\int_{c} \alpha=-\psi_{\alpha}(s),
\end{aligned}
$$

which implies that $\psi_{\alpha}(s)=0$.

Let $s_{1}, s_{2} \in S$ be two noncommuting elements. Then

$$
I\left[s_{1}, s_{2}\right]=\left[I\left(s_{1}\right), I\left(s_{2}\right)\right]=\left[s_{1}^{-1}, s_{2}^{-1}\right]
$$

is conjugate to $\left[s_{1}, s_{2}\right]$. Let $\gamma$ be a closed local geodesic representing the conjugacy class of $\left[s_{1}, s_{2}\right]$. It follows that $I \circ \gamma=\gamma$ (up to reparametrisation by a shift).

Let $\beta$ be a 1-form supported in a small ball such that $\int_{\gamma} \beta>0$, where $\gamma$ is as above. Let $\alpha=\beta+I^{*}(\beta)$. Then

$$
\begin{aligned}
\psi_{\alpha}\left[s_{1}, s_{2}\right] & =\int_{\gamma} \alpha=\int_{\gamma} \beta+I^{*}(\beta) \\
& =\int_{\gamma} \beta+\int_{I \circ \gamma} \beta=2 \int_{\gamma} \beta>0 .
\end{aligned}
$$

We thus constructed a nontrivial homogeneous quasimorphism which vanishes on the generating set $S$. A standard computation shows that it is Lipschitz with respect to the word norm associated with $S$ :

$$
\left|\psi_{\alpha}(g)\right|=\left|\psi_{\alpha}\left(s_{1} \cdots s_{n}\right)\right| \leq \sum_{i=1}^{n}\left|\psi_{\alpha}\left(s_{i}\right)\right|+\|g\|_{S} D=D\|g\|_{S} .
$$

where $D$ is the defect of $\psi_{\alpha}$. We finally obtain that

$$
0<n\left|\psi_{\alpha}\left(\left[s_{1}, s_{2}\right]\right)\right|=\left|\psi_{\alpha}\left(\left[s_{1}, s_{2}\right]^{n}\right)\right| \leq D\left\|\left[s_{1}, s_{2}\right]^{n}\right\|_{S}
$$

which shows that the translation length of $\left[s_{1}, s_{2}\right]$ is positive and hence the closed geodesic norm on $\Gamma_{x}$ is stably unbounded. In particular, it has infinite diameter.

Corollary 3.5 Let $(M, d)$ be as in Theorem 1.2. Let $s_{1}, s_{2} \in S$ be noncommuting elements represented by closed local geodesics. Then their commutator $\left[s_{1}, s_{2}\right]$ has positive translation length with respect to the closed geodesic norm.

Acknowledgements This work was partly funded by the Leverhulme Trust Research Project Grant RPG-2017159. MM is supported by the grant Sonatina 2018/28/C/ST1/00542 funded by Narodowe Centrum Nauki. MM and JK were partially supported by SFB 1085 "Higher Invariants" funded by Deutsche Forschungsgemeinschaft. 
Open Access This article is licensed under a Creative Commons Attribution 4.0 International License, which permits use, sharing, adaptation, distribution and reproduction in any medium or format, as long as you give appropriate credit to the original author(s) and the source, provide a link to the Creative Commons licence, and indicate if changes were made. The images or other third party material in this article are included in the article's Creative Commons licence, unless indicated otherwise in a credit line to the material. If material is not included in the article's Creative Commons licence and your intended use is not permitted by statutory regulation or exceeds the permitted use, you will need to obtain permission directly from the copyright holder. To view a copy of this licence, visit http://creativecommons.org/licenses/by/4.0/.

\section{References}

1. Ballmann, W., Thorbergsson, G., Ziller, W.: Closed geodesics and the fundamental group. Duke Math. J. 48(3), 585-588 (1981)

2. Bardakov, V., Shpilrain, V., Tolstykh, V.: On the palindromic and primitive widths of a free group. J. Algebra 285(2), 574-585 (2005)

3. Brandenbursky, M., Kędra, J., Shelukhin, E.: On the autonomous norm on the group of Hamiltonian diffeomorphisms of the torus. Commun. Contemp. Math. 20(2), 1750042 (2018)

4. Bridson, Martin R., Haefliger, André: Metric spaces of non-positive curvature. Grundlehren der Mathematischen Wissenschaften [Fundamental Principles of Mathematical Sciences], vol. 319. Springer-Verlag, Berlin (1999)

5. Calegari, D.: scl. MSJ Memoirs, vol. 20. Mathematical Society of Japan, Tokyo (2009)

6. Chinburg, T., Reid, A.W.: Closed hyperbolic 3-manifolds whose closed geodesics all are simple. J. Differ. Geom. 38(3), 545-558 (1993)

7. Gal, Ś.R., Kędra, J.: On bi-invariant word metrics. J. Topol. Anal. 3(2), 161-175 (2011)

8. Gal, Światosław R., Kędra, Jarek: Finite index subgroups in chevalley groups are bounded: an addendum to "on bi-invariant word metrics". arXiv:1808.06376 (2018)

9. Jones, K.N., Reid, A.W.: Geodesic intersections in arithmetic hyperbolic 3-manifolds. Duke Math. J. 89(1), 75-86 (1997)

10. Klingenberg, Wilhelm P. A.: Riemannian Geometry, volume 1 of De Gruyter Studies in Mathematics. Walter de Gruyter \& Co., Berlin, second edition (1995)

11. Margulis, G.A.: Discrete subgroups of semisimple Lie groups. Ergebnisse der Mathematik und ihrer Grenzgebiete (3) [Results in Mathematics and Related Areas (3)], vol. 17. Springer-Verlag, Berlin (1991)

12. Milnor, J.: A note on curvature and fundamental group. J. Differ. Geometry 2, 1-7 (1968)

13. Schwarz, A. S.: A volume invariant of coverings. In: Dokl. Akad. Nauk SSSR (N.S.), Vol 105, pp. 32-34 (1955)

Publisher's Note Springer Nature remains neutral with regard to jurisdictional claims in published maps and institutional affiliations. 